\title{
Non-Neoplastic Liver and Intrahepatic Bile Duct Disorder
}

National Cancer Institute

\section{Source}

National Cancer Institute. Non-Neoplastic Liver and Intrahepatic Bile Duct Disorder. NCI

Thesaurus. Code C53550.

A non-neoplastic disorder that affects the liver parenchyma and the intrahepatic bile ducts. Representative examples include hepatitis, cirrhosis, and cholangitis. 